\title{
Functionalized graphene - poly(vinyl alcohol) nanocomposites: Physical and dielectric properties
}

\author{
I. Tantis, G. C. Psarras* , D. Tasis \\ Department of Materials Science, School of Natural Sciences, University of Patras, Patras 26504, Hellas (Greece)
}

Received 29 July 2011; accepted in revised form 19 October 2011

\begin{abstract}
Chemically derivatized graphene/poly(vinyl alcohol) (PVA) nanocomposites were successfully fabricated by combination of solution processing and compression molding. SEM imaging combined with XRD measurements revealed that graphene platelets were fully incorporated into the polymer matrix after their chemical modification through adsorption of amphiphilic copolymer. The chemical functionalities onto the graphitic surface prevented particle aggregation and provided compatibility with the polymer matrix. Thermogravimetric analysis demonstrated enhanced thermal stability for the composites containing modified graphenes at loading above $1 \mathrm{wt} \%$. Differential scanning calorimetry thermograms showed that graphene nanoplatelets induced the crystallization of matrix with optimum loading at $2 \mathrm{wt} \%$. Dielectric spectroscopy measurements showed enhanced electrical permittivity for the graphene oxide/PVA system, compared to the one of graphene/copolymer/PVA. This could be attributed to the formation of an insulating coating between graphite inclusions and PVA because of the presence of the copolymer.
\end{abstract}

Keywords: polymer composites, nanocomposites, graphene, dielectric properties, thermal stability

\section{Introduction}

Polymer composites, filled by graphitic nanostructures, have attracted increased attention owing to their unique mechanical, electric and optical properties [1-3]. Nano-sized conductive fillers, including graphene nanoplatelets, can create a percolative network within the polymer matrix at a low weight fraction, while the presence of conductive nanoinclusions within a polymer matrix could alter the permittivity of the composite systems resulting in enhancement of their energy storing capability [4]. Nanoinclusions can be considered as a distributed network of nanocapacitors, which can be charged and discharged defining an energy storing process, at the nanoscale level [5]. Graphene platelets, prepared via natural graphite exfoliation, have been widely used as a conducting filler in the fabrication of conductive polymer composites [6-9]. Graphite has a layered crystal structure, in which the carbon atoms are strongly bonded on a two-dimensional network consisting of hexagons. Because the layers are bound by weak van der Waals forces, the idea of separating the bulk graphite into platelets consisting of few carbon layers having nanometer-scale thickness, or even into a single layer, has long been considered as the goal for obtaining super-strong and conductive polymer composites. Different types of graphite nanoplatelets, such as thermally expanded graphite $[10,11]$, graphene oxide (GO) $[12,13]$ and chemically modified graphene [14-17], have been used to make functional polymer composites. Chemical functionalization of graphene surface by either oxidation procedure or physical adsorption/grafting protocols have been found to be a feasible and effective means for improving the dispersion of graphenes in organic and/or aqueous media. In addi-

\footnotetext{
${ }^{*}$ Corresponding author, e-mail: G.C.Psarras@upatras.gr
} (c) BME-PT 
tion, the attached functional groups may enhance the interfacial interactions between the graphenes and the polymer matrix. The advantage of modifying the graphene surface by physical adsorption is that the structural integrity of the conjugated network remains unaltered, whereas formation of defects is observed after treatment of the graphitic nanostructures by oxidative conditions and/or grafting reactions.

To the best of our knowledge, there is no report on fabricating graphene/polymer composites by using noncovalently modified pristine graphene nanoplatelets. In addition, dielectric data such as dielectric permittivity, ac conductivity, and electric modulus have not been widely studied in graphene/ polymer composites. We show here a simple and environmentally friendly preparation of graphene/ PVA nanocomposites by incorporating amphiphilic block copolymer-modified graphenes into a PVA matrix using water as the compounding solvent. PVA is one of the most important commodity polymers due to its good mechanical and thermal properties. Its semicrystalline nature allows us to circumvent complexities of interpreting property changes associated with crystallization versus graphite addition. Here, we report a comparative study of PVA nanocomposites based on noncovalently modified graphene sheets, GO, and pristine graphite. The effect of graphene content on the physical and dielectric properties of PVA/graphene nanocomposites is investigated. The effect of filler's chemical functionalities as well as loading on various dielectric data (dielectric permittivity, ac conductivity, and electric modulus) was studied in detail.

\section{Experimental section}

\subsection{Materials}

Graphite with an average particle size of $45 \mu \mathrm{m}$ and a purity of $>95 \%$ was supplied from TIMCAL Ltd. (batch TIMREX KS44). Concentrated $\mathrm{H}_{2} \mathrm{SO}_{4}$, $\mathrm{K}_{2} \mathrm{~S}_{2} \mathrm{O}_{8}, \mathrm{P}_{2} \mathrm{O}_{5}$ and PVA with $M_{\mathrm{W}} \approx 145000$ were purchased from Sigma-Aldrich. The materials were directly used without further purification. The block

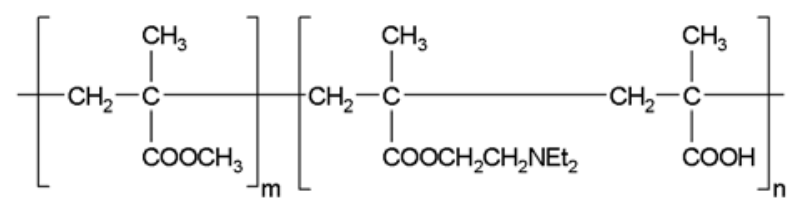

Figure 1. Chemical structure of the amphiphilic block copolymer copolymer, the chemical structure of which is shown in Figure 1, was synthesized by hydrolysis of the t-butylmethacrylate units of the precursor sample. The latter was kindly supplied by Prof. C. Tsitsilianis (Univ. of Patras).

\subsection{Preparation of graphene oxide}

GO was synthesized from graphite powder by a previously reported method [18]. The graphite powder $(15 \mathrm{~g})$ was put into an $80^{\circ} \mathrm{C}$ solution of concentrated $\mathrm{H}_{2} \mathrm{SO}_{4}(60 \mathrm{~mL}), \mathrm{K}_{2} \mathrm{~S}_{2} \mathrm{O}_{8}(7.8 \mathrm{~g})$, and $\mathrm{P}_{2} \mathrm{O}_{5}$ $(8.4 \mathrm{~g})$. After reaching the temperature, the mixture was left to cool down to room temperature over a period of $6 \mathrm{~h}$. The mixture was then carefully diluted with $300 \mathrm{~mL}$ of distilled water, filtered through $0.2 \mu \mathrm{m}$ PTFE membrane (Millipore), and washed on the filter until the $\mathrm{pH}$ of the rinsing water became neutral. The product was dried in vacuum at $55^{\circ} \mathrm{C}$ for 48 hours.

\subsection{Preparation of graphene/PVA composites}

The synthesis procedure for a typical well-dispersed graphene/PVA nanocomposite $(1.0 \mathrm{wt} \%)$ was as follows: Graphite powder $(33 \mathrm{mg})$ was dispersed in distilled water $(15 \mathrm{~mL})$ in an ultrasonic bath (Branson 2510 ) for $60 \mathrm{~min}$ at room temperature. Subsequently, an aqueous solution $(10 \mathrm{~mL})$ of the amphiphilic copolymer $(10 \mathrm{mg}$ ) was added to the graphite suspension. Sonication was continued for an extra 60 min to yield a stable black-colored suspension. Meanwhile, PVA ( $\sim 3.25 \mathrm{~g})$ was dissolved in distilled water $(100 \mathrm{~mL})$ at $90^{\circ} \mathrm{C}$ and the solution was subsequently cooled to room temperature. After the $\mathrm{PVA} / \mathrm{H}_{2} \mathrm{O}$ solution had cooled to around $50^{\circ} \mathrm{C}$, the graphene/copolymer aqueous dispersion was gradually added to the PVA solution and sonicated for an additional $30 \mathrm{~min}$ at room temperature. Finally, this homogeneous graphene/copolymer/PVA solution was poured into a Teflon Petri dish and kept at $60^{\circ} \mathrm{C}$ for film formation until its weight equilibrated. This film was peeled from of the substrate and was hot pressed at $200^{\circ} \mathrm{C}$ in order to eliminate the remaining air voids. A series of graphene/copolymer/ PVA nanocomposite films with different loadings of graphene nanoplatelets $(1,2,3$ and $5 \mathrm{wt} \%)$ were similarly prepared. In all the aforementioned samples, the ratio between graphene and copolymer was kept constant and was approximately $3: 1$. For comparison, GO/PVA composite films were pre- 
pared by the same protocol with loadings 1,2,3 and $5 \mathrm{wt} \%$, respectively, as well as one sample of pristine graphite/PVA film (1 wt \%). As a reference sample, neat PVA film was prepared by the hot casting technique.

\subsection{Characterization}

Fourier transform infrared spectra (FTIR) of graphite material were obtained on a EXCALIBUR FTS300 (Digilab) spectrometer by the KBr pellet method. The thermal transitions of the samples were investigated by Differential Scanning Calorimetry (DSC) using a DSC Q200 (TA instruments). The experiments were carried out in nitrogen atmosphere using about $7 \mathrm{mg}$ sample sealed in aluminium pans. The samples were heated from room temperature to $250^{\circ} \mathrm{C}$. The heating rate was $10^{\circ} \mathrm{C} / \mathrm{min}$ in all cases. Thermogravimetric analysis (TGA) was performed on a TGA Q500 (TA instruments) at a heating rate of $10^{\circ} \mathrm{C} / \mathrm{min}$ in a nitrogen atmosphere. Scanning electron microscopy (LEO SUPRA $35 \mathrm{VP}$ ) was used to observe the edge as well as the top surfaces of the graphene/PVA nanocomposite films. The samples were coated with gold before analysis. The crystalline structure of the samples was analyzed with an X-ray powder diffractometer (Bruker D8 Advance) using $\mathrm{Cu} \mathrm{K} \mathrm{K}_{\alpha}$ radiation $(\lambda=0.15418 \mathrm{~nm})$. The XRD measurements were carried out in the $2 \theta$ angle with the range of 5-35 . Electrical characterization was carried out by means of Broadband Dielectric Spectroscopy (BDS) in the frequency range of $0.1 \mathrm{~Hz}$ to $1 \mathrm{MHz}$, using an Alpha-N Frequency Response Analyser, supplied by Novocontrol. A BDS-1200, parallel-plate capacitor with two gold-plated electrodes system, supplied also by Novocontrol, was used as dielectric test cell. The electrically tested samples were in the form of a circular disk with diameter $12 \mathrm{~mm}$ and thickness $0.5 \mathrm{~mm}$. The amplitude of the time-varying applied voltage was kept constant at $1000 \mathrm{mV}$. Temperature was controlled via a Novotherm system (Novocontrol). Measurements were performed in the range of 30 to $120^{\circ} \mathrm{C}$. Isothermal scans were carried out for each sample with a temperature step of $10^{\circ} \mathrm{C}$. The dielectric cell was electrically shielded and both instruments were interfaced to a PC for simultaneous control and data acquisition.

\section{Results and discussion}

The oxidation of graphite can be confirmed by infrared spectroscopy. The FTIR spectrum (data not shown) of graphene oxide showed a broad band at about $3450 \mathrm{~cm}^{-1}$ which is due to vibrations of $\mathrm{O}-\mathrm{H}$ bonds. The peak at about $1100 \mathrm{~cm}^{-1}$ indicates the existence of the oxygen-containing functional groups on GO, such as epoxide, which were formed during oxidation reactions [19].

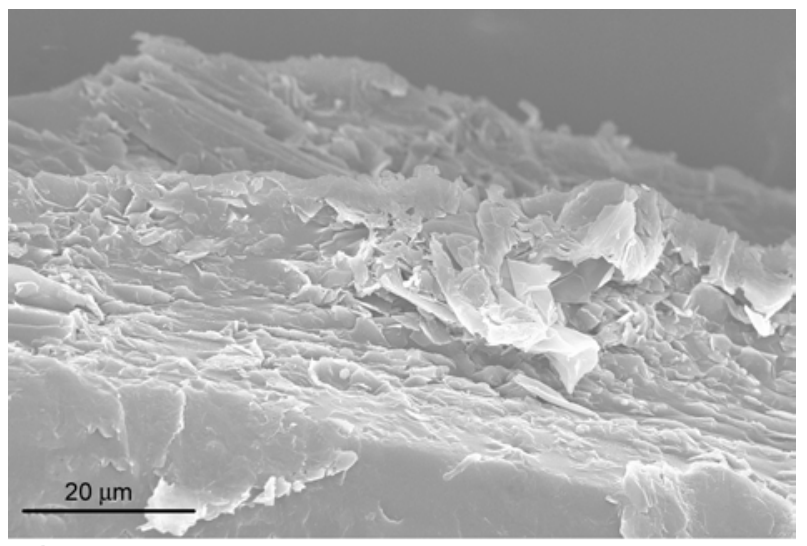

a)

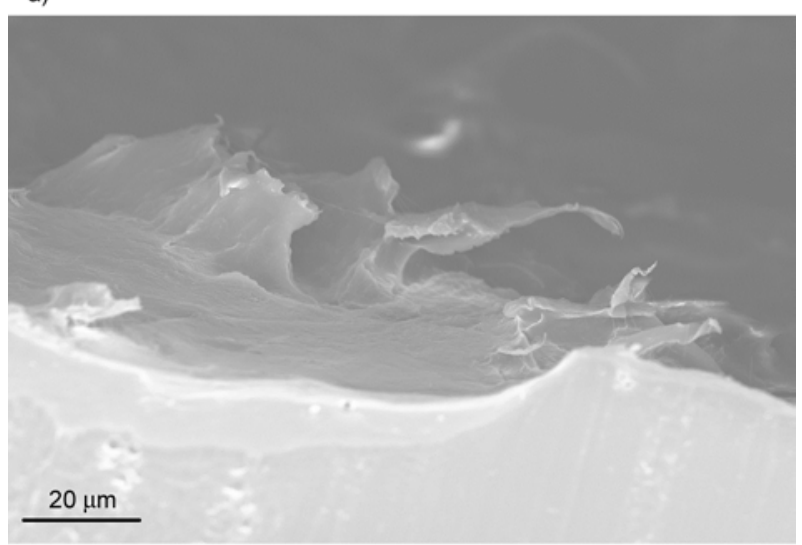

b)

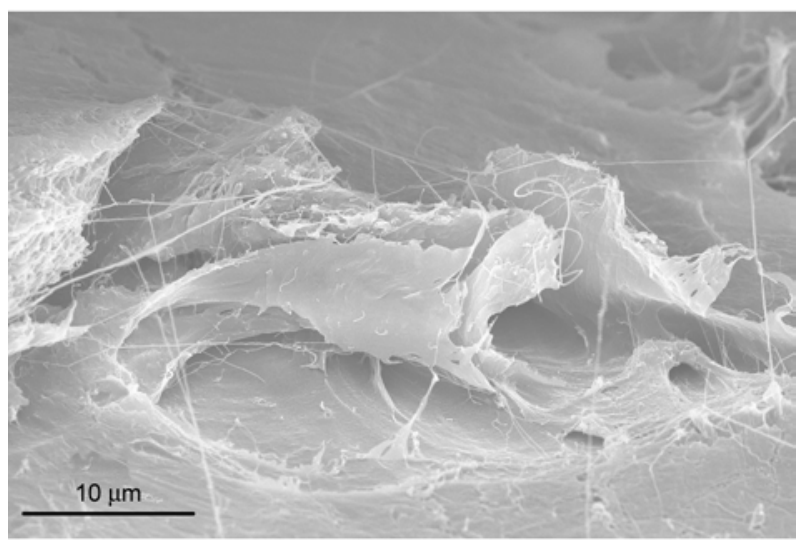

c)

Figure 2. SEM images of composite films ( $1 \mathrm{wt} \%$ graphene loading). Cross sections of pristine- (a), GO- (b) and copolymer-modified graphene-polymer composites (c) 
In order to investigate the influence of the graphene chemical functionalities on the morphology, as well as the dielectric properties of PVA-based composites, GO and copolymer-modified graphene were blended with the polymer matrix by solution mixing/hot casting process. SEM images (Figure 2) show cross sections of composite films containing $1 \mathrm{wt} \%$ pristine or modified nanographite. As shown in image a), pristine graphite is poorly dispersed in the composite, with agglomerates at length scales of tens of microns. Poor dispersion of unmodified graphite in solution-cast samples was expected and was a driving force for the use of chemically modified graphite, such as graphene oxide and surfactantmodified material. Characterization via SEM of the corresponding samples (images b) and c)) showed much better distribution of graphite particles homogeneously covered by the polymer. As graphene oxide does, our copolymer-modified graphene nanoplatelets can be homogeneously integrated within hydrophilic PVA matrix. The presence of ionic moieties, such as alkylamino- and carboxylates in the polymer backbone result in enhanced interfacial interactions between the filler and the matrix, whereas its amphiphilic character is responsible for the efficient exfoliation of graphene nanoplatelets by noncovalent approach. This property makes copolymer-modified graphenes a potential candidate as filler material in functional polymer composites.

The structures of GO powder, the neat polymer matrix as well as the composite films was studied by XRD (Figure 3). The XRD diffraction peak of GO sheets is appeared at $26.7^{\circ}$, implying that its $\mathrm{d}$-spacing resembles the one of pristine graphite.

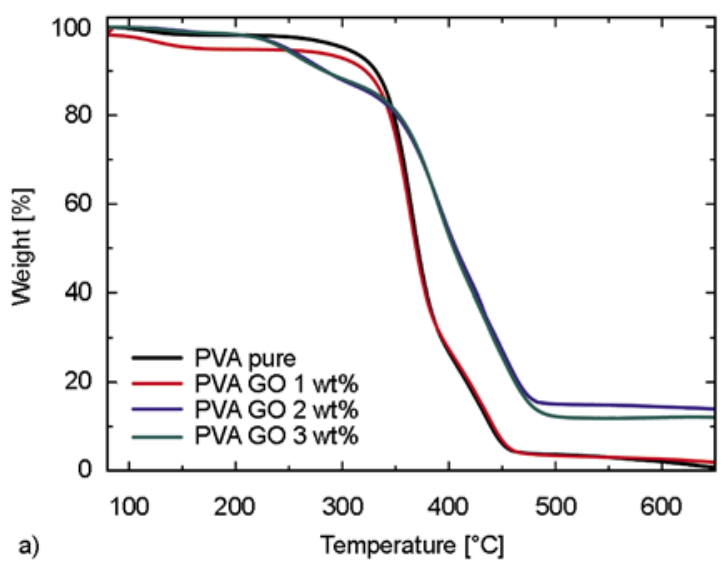

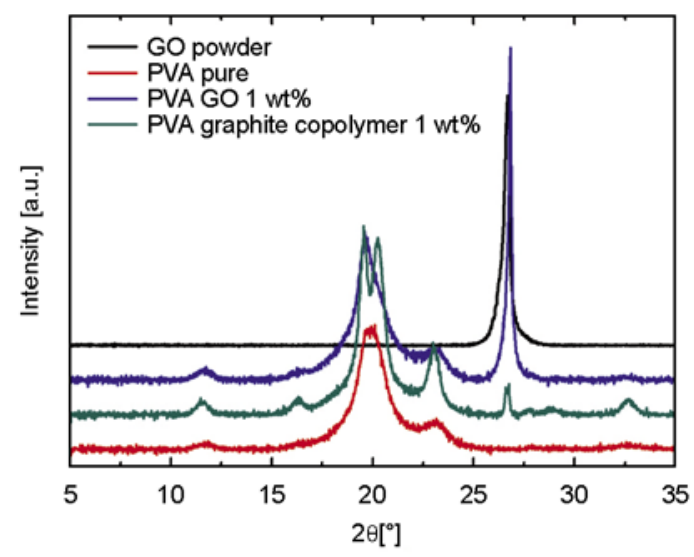

Figure 3. XRD patterns of GO powder, PVA matrix, $1 \mathrm{wt} \%$ $\mathrm{GO} / \mathrm{PVA}$ and $1 \mathrm{wt} \%$ graphite/copolymer/PVA

The lack of graphene expansion can be attributed to the mild oxidative treatment of the starting material. Diffraction peaks of neat PVA film are found at $19.9^{\circ}$ (main), as well as $11.7,23.1$, and $32.7^{\circ}$ (minor) [13]. The XRD pattern of the $1 \mathrm{wt} \% \mathrm{GO} / \mathrm{PVA}$ composite film has all the peaks assigned to both GO and the matrix. This means that GO sheets were not individually dispersed in PVA matrix but rather in the form of few-layer GO platelets. On the contrary, the XRD profile of the $1 \mathrm{wt} \%$ graphite/copolymer/ PVA composite film shows a peak of diminished intensity at $26.7^{\circ}$. This result indicated that graphite has been efficiently exfoliated within the PVA matrix, through its noncovalent functionalization with the amphiphilic copolymer. In addition, the crystalline structure of the matrix was slightly affected due to the incorporation of the graphene platelets.

The thermal stability of the composite materials was assessed by thermogravimetric analysis (TGA) in inert atmosphere. Figure 4a showed that the onset temperature for neat PVA degradation was about

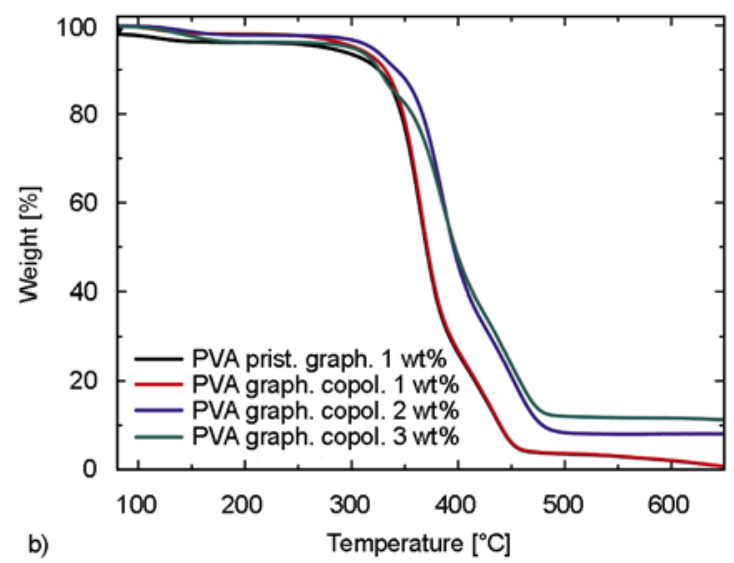

Figure 4. TGA profiles of: (a) neat matrix and GO/PVA (1, 2 and $3 \mathrm{wt} \%$ ), (b) $1 \mathrm{wt} \%$ pristine graphite/PVA and graphene/ copolymer/PVA (1, 2 and $3 \mathrm{wt} \%)$ 
$300^{\circ} \mathrm{C}$, which was attributed to main-chain pyrolysis. At about $450^{\circ} \mathrm{C}$, the total amount of polymer was seemed to be pyrolyzed. From the TGA profiles of the composite samples, it was shown that the ones containing $1 \mathrm{wt} \%$ pristine graphite, $1 \mathrm{wt} \% \mathrm{GO}$ and $1 \mathrm{wt} \%$ copolymer-modified graphene exhibited similar thermal stability, as that of neat matrix. In the TGA curves of the 2 and $3 \mathrm{wt} \%$ GO-based composites (Figure 4), there were two steps in the degradation of the composites: The first step, from 200 to $280^{\circ} \mathrm{C}$, was attributed to the elimination of the oxygen-containing groups onto the oxidized graphene nanoplatelets; the second step, roughly from 350 to $480^{\circ} \mathrm{C}$, which is the degradation of the polymer, has mildly shifted to a higher temperature range with respect to the neat polymer matrix [13]. This indicates that there is a strong interaction between PVA and GO nanoplatelets at the interface, and, because of this, the mobility of the polymer chains near the interface has decreased. Similarly, concerning the graphene/copolymer/PVA samples, enhanced thermal stability was observed for the composites bearing 2 and $3 \mathrm{wt} \%$ graphene, comparing to the neat matrix.

To confirm the strong interaction of graphene nanostructures with the polymer matrix, differential scanning calorimetry (DSC) was used. DSC thermograms (Figure 5) obtained on heating scans showed that the integrated area of the melting transition $\left(\Delta H_{\mathrm{m}}\right)$ of the samples, containing either GO or copolymer-modified graphene nanoplatelets, reached a maximum at $2 \mathrm{wt} \%$ filler loading. In this graphene weight fraction, it was estimated that the relative crystallinity of the copolymer-modified graphene/PVA sample was increased by about

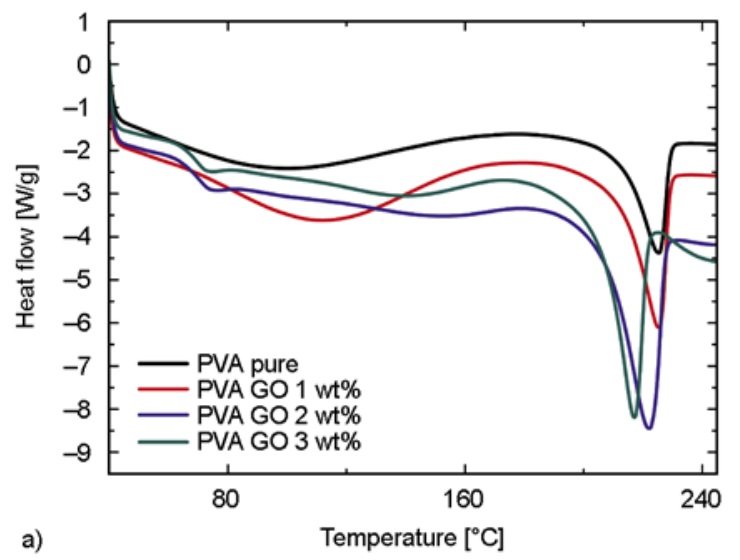

$150 \%$, compared to the one of neat matrix. Similar results have been demonstrated for CNT/PVA composite films [20]. This crystallinity increase indicates that the polymer chains were indeed immobilized by hydrophobic and/or hydrogen bonding interactions with the graphene nanoplatelets, an effect which could support the slightly enhanced thermal stability of the composites with filler loading $>1 \mathrm{wt} \%$ (see TGA data). In a recent study [13], researchers observed no deviations of crystallinity values in GO/PVA composites, yet, the filler loading of the studied samples was between 0.3 and $0.7 \mathrm{wt} \%$. Overall, the order of relative crystallinity in our samples (at $1 \mathrm{wt} \%$ graphene loading) was: copolymer-modified graphene $>\mathrm{GO}>$ neat matrix $>$ pristine graphite. The fact that the sample of pristine graphite $1 \% \mathrm{wt} / \mathrm{PVA}$ exhibited a $15 \%$ decrease in crystallinity compared to neat PVA could be attributed to the inhomogeneous dispersion of graphite aggregates into the matrix. The melting endothermic peak of neat matrix (at about $225^{\circ} \mathrm{C}$ ) was slightly decreased in the composites. This could be attributed to the relatively smaller crystal size of PVA, due to the intercalation of graphene platelets.

The interest in graphene-based composites stems from their potential high electrical conductivity and permittivity. Dielectric data can be analyzed by means of different formalisms, such as dielectric permittivity, ac conductivity, and electric modulus. Although, all three formalisms can be employed on the description and analysis of the occurring electrical effects, a certain formalism can be proved more effective in extracting information regarding the occurring physical mechanisms under specific con-

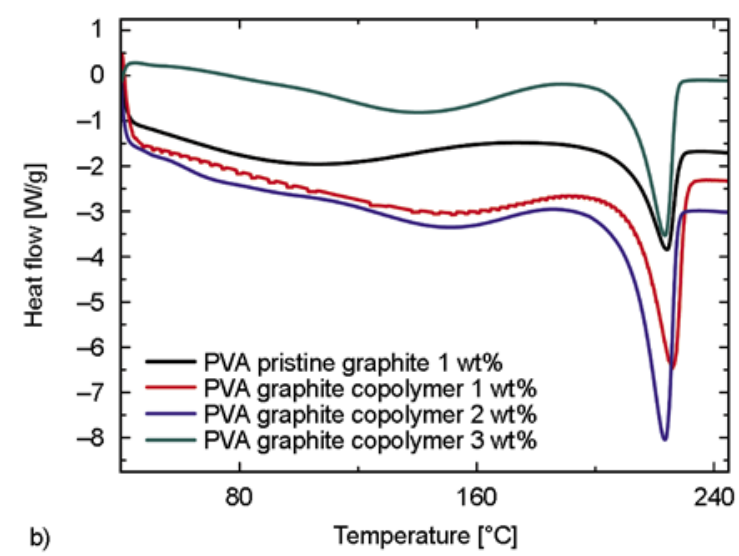

Figure 5. DSC thermograms of: (a) neat matrix and GO/PVA (1, 2 and $3 \mathrm{wt} \%$ ), (b) $1 \mathrm{wt} \%$ pristine graphite/PVA, and graphene/copolymer/PVA (1, 2 and $3 \mathrm{wt} \%)$ 
ditions. In the present study, experimental data were firstly expressed via the real and imaginary part of dielectric permittivity and then transformed into electric modulus formalism [21-23]. Figure 6a depicts the variation of real part of dielectric permittivity $\left(\varepsilon^{\prime}\right)$ with frequency for all types of the produced composites at constant temperature $\left(30^{\circ} \mathrm{C}\right)$ and nanofiller content $(1 \mathrm{wt} \%)$. At low frequencies, permittivity attained higher values, in all cases, which diminished rapidly with frequency. This is reasonable since - in the low frequency region - the alternation of the field is slow, providing thus sufficient time to permanent and induced dipoles to align themselves according to the applied field, leading to enhanced polarization. Neat PVA sample and graphene/copolymer/PVA nanocomposite exhibited almost identical values, while the pristine graphite/PVA sample exhibited slightly higher values than neat matrix. On the other hand, pronounced alteration of permittivity was evident in the spectrum of GO/PVA nanocomposite. Enhanced values of $\left(\varepsilon^{\prime}\right)$, especially at low frequencies, can be attributed to increased conductivity, and/or interfacial polarization, and/or electrode polarization. Electrode polarization is related to the build up of space charges at the specimen-electrode interfaces and is characterized by very high values of both real and imaginary part of dielectric permittivity [2123]. Examined samples were tested under identical experimental conditions, having similar geometrical characteristics and composition. Thus, if electrode polarization was the prevalent effect in a certain type of nanocomposite, should be also the prevalent tendency to all other types. Under this point of view, the higher values of $\left(\varepsilon^{\prime}\right)$ could be attributed to enhanced conductivity and interfacial polarization (IP). IP results from the accumulation of unbounded charges at the interfaces of the constituents, where they form large dipoles. Its intensity is connected to the extent of the existing interfacial area within the composite system, giving thus indirect evidence of the achieved distribution of nanoinclusions [24]. Therefore, the low values of $\left(\varepsilon^{\prime}\right)$ in graphene/copolymer/PVA and pristine graphite/ PVA spectra could be assigned to lower overall conductivity and less satisfactory distribution of nanofiller, respectively. Figures $6 \mathrm{~b}$ and $6 \mathrm{c}$, respectively, present the variation of permittivity with respect of frequency for the graphene/copolymer/PVA and
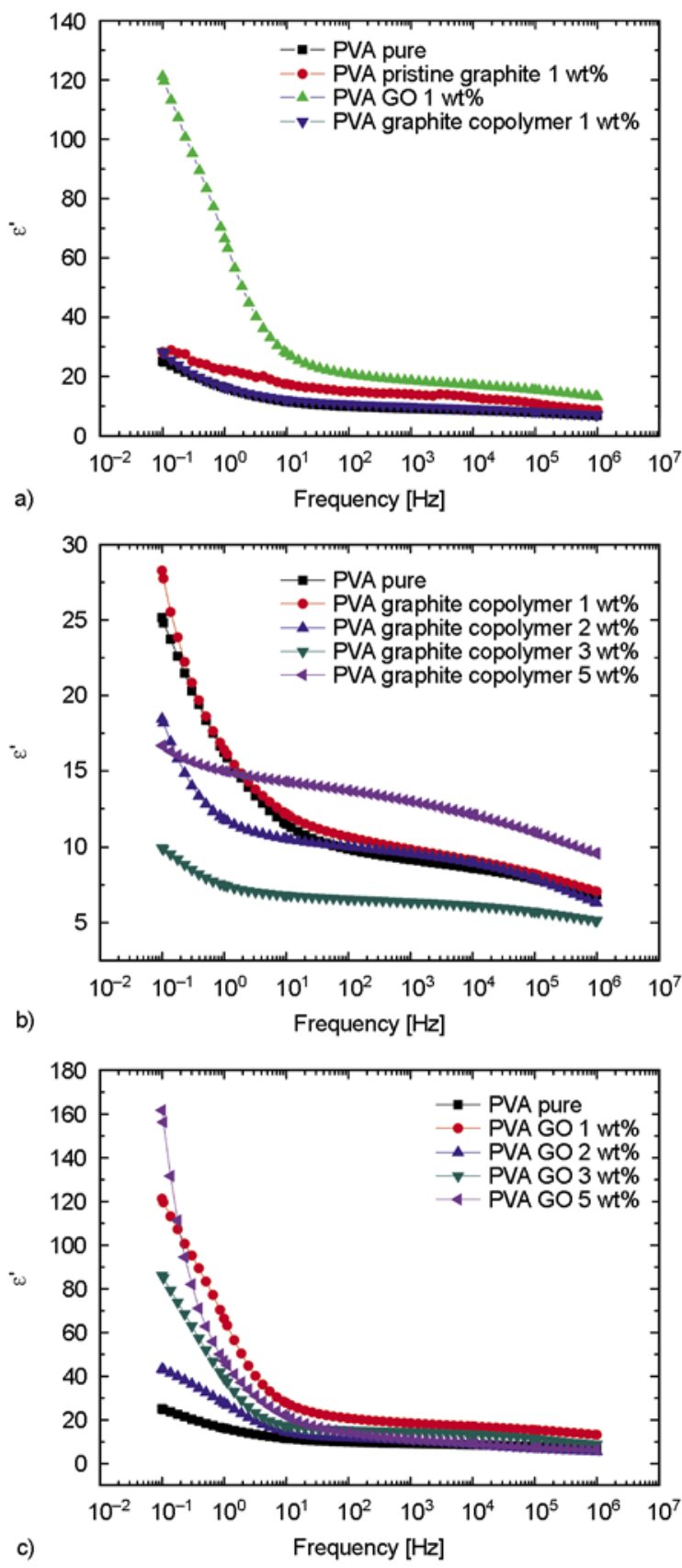

Figure 6. Variation of real part of dielectric permittivity $\left(\varepsilon^{\prime}\right)$ with frequency for: (a) all types of the produced composites at constant temperature $\left(30^{\circ} \mathrm{C}\right)$ and at constant filler content ( $1 \mathrm{wt} \%)$, (b) composites with copolymer-modified graphite varying the filler content, and (c) composites with GO nanoplatelets varying the filler content. In all cases pure PVA spectrum is given for comparison.

GO/PVA systems, in various filler loadings. The graphene/copolymer/PVA system displayed a tendency for diminished values of permittivity with filler content, which could be attributed to the formation of an insulating coating between graphite inclusions and PVA because of the presence of the 
copolymer. On the contrary, in the spectra of $\mathrm{GO} /$ PVA samples, a systematic increase of $\left(\varepsilon^{\prime}\right)$ with filler content was recorded for all samples, with the unexpected exception of PVA GO 1\% wt. Since polarization and stored energy is directly proportional to permittivity, Figure 6 implies that - under the specific processing conditions - GO nanofiller provides the best, from all the examined cases, electrical enhancement.

The variation of imaginary part of electric modulus $\left(M^{\prime \prime}\right)$ versus frequency for the PVA samples containing $2 \mathrm{wt} \%$ fraction of copolymer-modified graphite and GO nanoplatelets, at various temperatures, is depicted in Figure 7. The imaginary part of electric modulus $\left(M^{\prime \prime}\right)$ vs frequency spectra revealed two relaxation mechanisms. The one recorded at high frequencies was attributed to glass transition of the polymer matrix ( $\alpha$-mode) and the slower one to (IP). The $\alpha$-mode, which is present also in the dielectric spectrum of pure PVA, is related to motions of large parts of the main polymer chain. Dielectric loss peak position shifts to higher frequencies with increasing temperature, since thermal agitation facilitates the orientation of polar parts. Further, in the case of GO/PVA systems, loss peaks at constant temperature were recorded at relatively higher frequencies in comparison to the same set of peaks of the graphene/copolymer/PVA systems. Shift of $\alpha$-mode's loss peak to higher frequency has been considered as an indication for lowering of glass transition temperature, because of weak or moderate interactions between host medium and filler [25]. Thus, the graphene/copolymer/PVA systems should be characterized by higher $T_{\mathrm{g}}$, which is also supported by DSC spectra. In the low frequency range spectra of Figure 7, a second peak or

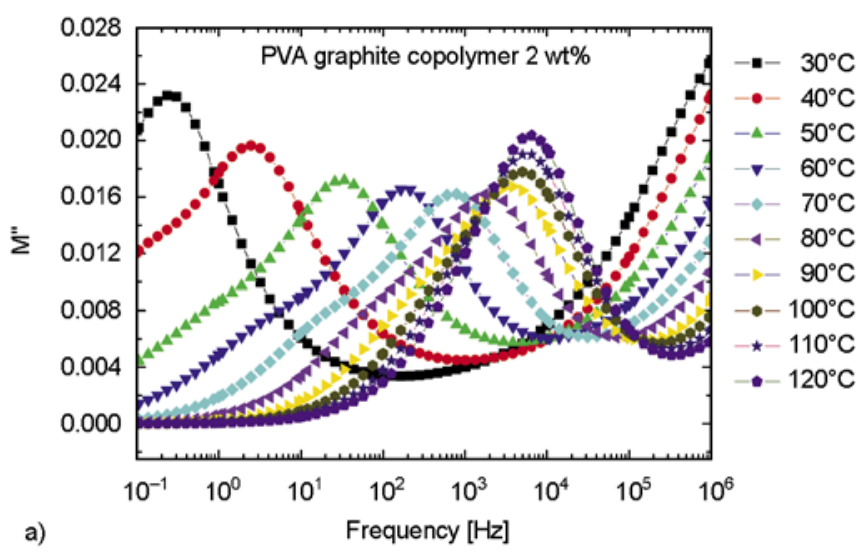

'hump' was observed, which corresponds to a slower process, characterized by enhanced relaxation time. As already mentioned, this process was assigned to IP. The latter appears to electrically heterogeneous systems due to the accumulation of charges at the system's interface. The presence of these charges originates from the stage of specimens' preparation. At the interface, the formed dipoles try to follow the alternation of the applied field and because of their inertia a relaxation process occurs [26].

Relaxation dynamics is related to the temperatureinduced dielectric loss peak shift. Figure 8 depicts the temperature dependence of the loss peak frequency for the GO/PVA and copolymer/PVA systems for the main relaxation process ( $\alpha$-mode). In all cases the recorded temperature dependence deviates from a pure Arrhenius behaviour, and can be better described via the Vogel-Fulcher-Tamann (VFT) equation, according to which relaxation rate increases rapidly at lower temperatures, because of the reduction of free volume. VFT can be expressed as Equation (1):

$f_{\max }=f_{0} \exp \left(-\frac{A T_{0}}{T-T_{0}}\right)$

where $f_{0}$ is a pre-exponential factor, $A$ a constant (being a measure of activation energy), and $T_{0}$ the Vogel temperature or ideal glass transition temperature. Parameters resulted via fitting data with Equation (1) are listed in Table 1. Note that the parameter $T_{0}$ attains higher values in the systems with the copolymer, being in accordance with the obtained results from different experimental techniques, mentioned previously. In the literature it is well accepted that the glass to rubber transition follows the VFT equation [24, 27, 28], thus Figure 8 gives

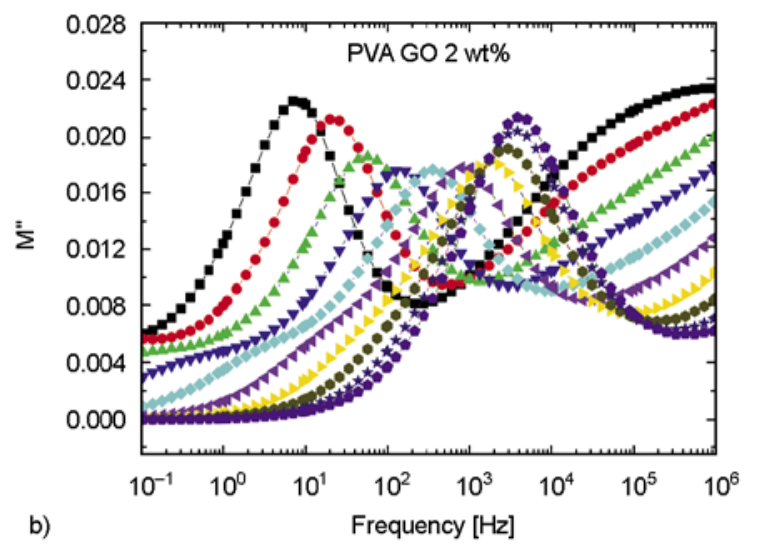

Figure 7. Variation of imaginary part of electric modulus $\left(M^{\prime \prime}\right)$ versus frequency for the PVA samples containing 2 wt\% fraction of: (a) copolymer-modified graphite and (b) GO nanoplatelets, at various temperatures 
Table 1. Fitting parameters of VFT equation for $\alpha$-mode of the examined systems

\begin{tabular}{|c|c|c|c|c|c|}
\hline Sample & $\mathbf{A}$ & $\mathbf{T}_{0}[\mathbf{K}]$ & Sample & A & $\mathbf{T}_{0}[\mathbf{K}]$ \\
\hline PVA GO $1 \% \mathrm{wt}$ & 6.6 & 220.4 & PVA Graphite Copolymer 1\% wt & 4.3 & 220.4 \\
\hline PVA GO $2 \% \mathrm{wt}$ & 6.7 & 207.2 & PVA Graphite Copolymer $2 \% \mathrm{wt}$ & 2.8 & 258.2 \\
\hline PVA GO 3\% wt & 6.3 & 213.7 & PVA Graphite Copolymer 3\% wt & 2.4 & 264.2 \\
\hline PVA GO 5\% wt & 6.1 & 217.8 & PVA Graphite Copolymer 5\% wt & 1.8 & 287.5 \\
\hline
\end{tabular}
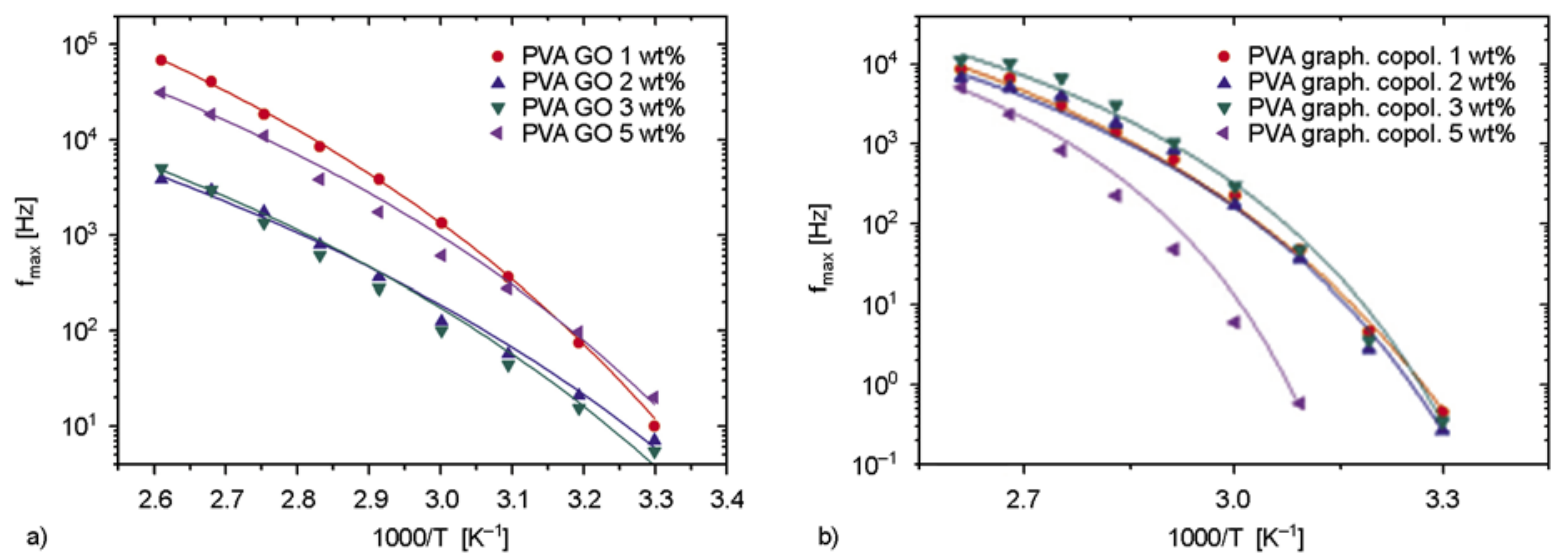

Figure 8. Loss peak position as a function of the reciprocal temperature for the: (a) GO/PVA systems and (b) the graphite/copolymer/PVA systems

secondary support in assigning the main relaxation mechanism of Figure 8 as $\alpha$-mode.

Figure 9 shows the variation of ac conductivity with frequency for the graphene/copolymer/PVA and $\mathrm{GO} / \mathrm{PVA}$ nanosystems at $30^{\circ} \mathrm{C}$. Conductivity appears to be frequency dependent. In the low frequency range, conductivity tends to acquire constant values approaching its dc value, while after a critical value varies exponentially with frequency $[29,30]$. This type of behaviour is common in disordered solids, appears to be in accordance with the so-called 'ac universality law', and is considered as a strong indication for charge migration via the hopping mechanism $[25,30]$. GO/PVA systems

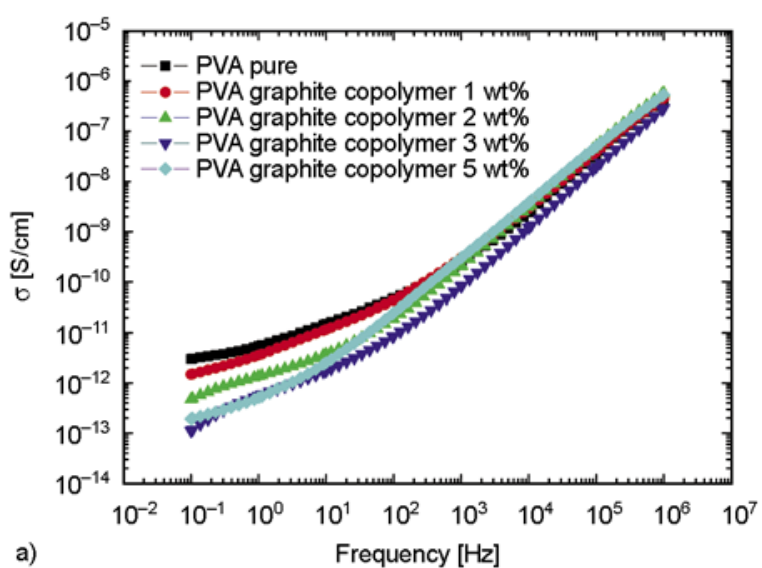

exhibited relatively higher values of conductivity, which was in general altered with GO loading. On the contrary, conductivity of graphene/copolymer/ PVA systems was diminished with the graphene weight fraction. The latter is in accordance with the variation of permittivity with copolymer-modified graphene content (Figure 6b), providing extra support to the assumption that treating of graphenes with the copolymer results in better distribution and mutual isolation of conductive nanoinclusions. In insulating matrix-conductive inclusions composites the area between adjacent isolated conductive elements appears to be crucial. The local contact regions, between conductive inclusions, control the

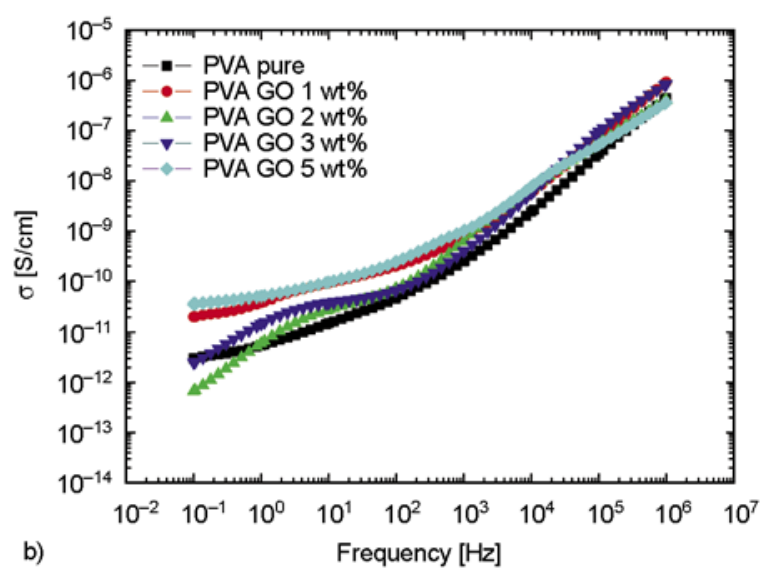

Figure 9. Variation of ac conductivity with frequency for the: (a) graphene/copolymer/PVA and (b) GO/PVA nanosystems, at $30^{\circ} \mathrm{C}$ 
overall conductance of the system. Abutting conductive elements separated by polymer chains restrict the conductivity values [31,32]. Copolymer produces an electrical insulation of graphene inclusions, leading to lower values of conductivity. Finally, it should be stated that in all studied specimens, conductivity was not altered abruptly, implying that the transition from insulating to conductive behaviour has not been achieved at the examined set of composites.

\section{Conclusions}

In this study, PVA polymer was blended with either graphene oxide or functionalized graphene sheets. A surfactant-like block copolymer was employed as exfoliation/dispersion agent of the graphene nanoplatelets within PVA matrix through solution processing and compression molding. Concerning the graphene/PVA samples, enhanced thermal stability was observed for the composites bearing graphene loading above $1 \mathrm{wt} \%$, comparing to the neat matrix. DSC thermograms showed that the integrated area of the melting transition $\left(\Delta H_{\mathrm{m}}\right)$ of the samples, containing either $\mathrm{GO}$ or copolymer-modified graphene nanoplatelets, reached a maximum at $2 \mathrm{wt} \%$ filler loading. Dielectric measurements revealed that graphene/copolymer/PVA system displayed a tendency for diminished values of permittivity $\left(\varepsilon^{\prime}\right)$ with filler content, which could be attributed to the formation of an insulating coating between graphite inclusions and PVA because of the presence of the copolymer. On the contrary, in the spectra of GO/ PVA samples, a systematic increase of $\left(\varepsilon^{\prime}\right)$ with filler content was recorded.

\section{References}

[1] Spitalsky Z., Tasis D., Papagelis K., Galiotis C.: Carbon nanotube-polymer composites: Chemistry, processing, mechanical and electrical properties. Progress in Polymer Science, 35, 357-401 (2010). DOI: $10.1016 /$ j.progpolymsci.2009.09.003

[2] Cai D., Song M.: Recent advance in functionalized graphene/polymer nanocomposites. Journal of Materials Chemistry, 20, 7906-7915 (2010).

DOI: $10.1039 / \mathrm{c} 0 \mathrm{jm} 00530 \mathrm{~d}$

[3] Kim H., Abdala A. A., Macosko C. W.: Graphene/ polymer nanocomposites. Macromolecules, 43, 65156530 (2010).

DOI: $10.1021 / \mathrm{ma1} 100572 \mathrm{e}$
[4] Zhang L. L., Zhao S., Tian X. N., Zhao X. S.: Layered graphene oxide nanostructures with sandwiched conducting polymers as supercapacitor electrodes. Langmuir, 26, 17624-17628 (2010).

DOI: $10.1021 / 1 \mathrm{a} 103413 \mathrm{~s}$

[5] Psarras G. C.: Nanodielectrics: An emerging sector of polymer nanocomposites. Express Polymer Letters, 2, 460 (2008).

DOI: 10.3144/expresspolymlett.2008.55

[6] Wakabayashi K., Pierre C., Dikin D. A., Ruoff R. S., Ramanathan T., Brinson L. C., Torkelson J. M.: Polymer-graphite nanocomposites: Effective dispersion and major property enhancement via solid-state shear pulverization. Macromolecules, 41, 1905-1908 (2008). DOI: $\underline{10.1021 / \mathrm{ma} 071687 \mathrm{~b}}$

[7] Du X. S., Xiao M., Meng Y. Z.: Facile synthesis of highly conductive polyaniline/graphite nanocomposites. European Polymer Journal, 40, 1489-1493 (2004). DOI: 10.1016/j.eurpolymj.2004.02.009

[8] Wu H., Zhao W., Hu H., Chen G.: One-step in situ ball milling synthesis of polymer-functionalized graphene nanocomposites. Journal of Materials Chemistry, 21, 8626-8632 (2011).

DOI: $10.1039 / \mathrm{c} 1 \mathrm{jm} 10819 \mathrm{k}$

[9] Biswas S., Fukushima H., Drzal L. T.: Mechanical and electrical property enhancement in exfoliated graphene nanoplatelet/liquid crystalline polymer nanocomposites. Composites Part A: Applied Science and Manufacturing, 42, 371-375 (2011). DOI: $10.1016 /$ j.compositesa.2010.12.006

[10] Raghu A. V., Lee Y. R., Jeong H. M., Shin C. M.: Preparation and physical properties of waterborne polyurethane/functionalized graphene sheet nanocomposites. Macromolecular Chemistry and Physics, 209, 2487-2493 (2008).

DOI: $10.1002 / \mathrm{macp} .200800395$

[11] Ansari S., Giannelis E. P.: Functionalized graphene sheet-poly(vinylidene fluoride) conductive nanocomposites. Journal of Polymer Science Part B: Polymer Physics, 47, 888-897 (2009). DOI: $10.1002 /$ polb.21695

[12] Dutta K., De S. K.: Electrical conductivity and optical properties of polyaniline intercalated graphite oxide nanocomposites. Journal of Nanoscience and Nanotechnology, 7, 2459-2465 (2007). DOI: $10.1166 /$ jnn.2007.429

[13] Liang J., Huang Y., Zhang L., Wang Y., Ma Y., Guo T., Chen Y.: Molecular-level dispersion of graphene into poly(vinyl alcohol) and effective reinforcement of their nanocomposites. Advanced Functional Materials, 19, 2297-2302 (2009). DOI: $10.1002 / \mathrm{adfm} .200801776$

[14] Stankovich S., Dikin D. A., Dommett G. H. B., Kohlhaas K. M., Zimney E. J., Stach E. A., Piner R. D., Nguyen S. T., Ruoff R. S.: Graphene-based composite materials. Nature, 442, 282-286 (2006).

DOI: $10.1038 /$ nature 04969 
[15] Liu N., Luo F., Wu H., Liu Y., Zhang C., Chen J.: Onestep ionic-liquid-assisted electrochemical synthesis of ionic-liquid-functionalized graphene sheets directly from graphite. Advanced Functional Materials, 18, 1518-1525 (2008).

DOI: $10.1002 / \mathrm{adfm} .200700797$

[16] Xu Y., Wang Y., Liang J., Huang Y., Ma Y., Wan X., Chen Y.: A hybrid material of graphene and poly $(3,4-$ ethyldioxythiophene) with high conductivity, flexibility, and transparency. Nano Research, 2, 343-348 (2009). DOI: $10.1007 / \mathrm{s} 12274-009-9032-9$

[17] Liang J., Xu Y., Huang Y., Zhang L., Wang Y., Ma Y., Li F., Guo T., Chen Y.: Infrared-triggered actuators from graphene-based nanocomposites. The Journal of Physical Chemistry C, 113, 9921-9927 (2009). DOI: 10.1021/jp901284d

[18] Kovtyukhova N. I., Ollivier P. J., Martin B. R., Mallouk T. E., Chizhik S. A., Buzaneva E. V., Gorchinskiy A. D.: Layer-by-layer assembly of ultrathin composite films from micron-sized graphite oxide sheets and polycations. Chemistry of Materials, 11, 771-778 (1999). DOI: $10.1021 / \mathrm{cm} 981085 \mathrm{u}$

[19] Zheng G., Wu J., Wang W., Pan C.: Characterizations of expanded graphite/polymer composites prepared by in situ polymerization. Carbon, 42, 2839-2847 (2004). DOI: $10.1016 /$ j.carbon.2004.06.029

[20] Ryan K. P., Cadek M., Nicolosi V., Blond D., Ruether M., Armstrong G., Swan H., Fonseca A., Nagy J. B., Maser W. K., Blau W. J., Coleman J. N.: Carbon nanotubes for reinforcement of plastics? A case study with poly(vinyl alcohol). Composites Science and Technology, 67, 1640-1649 (2007).

DOI: 10.1016/j.compscitech.2006.07.006

[21] Tsangaris G. M., Psarras G. C., Kouloumbi N.: Electric modulus and interfacial polarization in composite polymeric systems. Journal of Materials Science, 33, 2027-2037 (1998).

DOI: $10.1023 / \mathrm{A}: 1004398514901$

[22] Gatos K. G., Martínez Alcázar J. G., Psarras G. C., Thomann R., Karger-Kocsis J.: Polyurethane latex/ water dispersible boehmite alumina nanocomposites: Thermal, mechanical and dielectrical properties. Composites Science and Technology, 67, 157-167 (2007). DOI: $\underline{10.1016 / \text { j.compscitech.2006.07.025 }}$
[23] Psarras G. C., Gatos K. G., Karahaliou P. K., Georga S. N., Krontiras C. A., Karger-Kocsis J.: Relaxation phenomena in rubber/layered silicate nanocomposites. Express Polymer Letters, 1, 837-845 (2007).

DOI: 10.3144/expresspolymlett.2007.116

[24] Kalini A., Gatos K. G., Karahaliou P. K., Georga S. N., Krontiras C. A., Psarras G. C.: Probing the dielectric response of polyurethane/alumina nanocomposites. Journal of Polymer Science Part B: Polymer Physics, 48, 2346-2354 (2010).

DOI: $10.1002 /$ polb.22120

[25] Psarras G. C.: Conductivity and dielectric characterization of polymer nanocomposites. in 'Physical properties of polymer nanocomposites’ (eds.: Tjong S. C., Mai Y. W.) Woodhead, Cambridge, 31-69 (2010).

[26] Mitra S., Mondal O., Saha D. R., Datta A., Banerjee S., Chakravorty D.: Magnetodielectric effect in graphenePVA nanocomposites. Journal of Physical Chemistry C, 115, 14285-14289, (2011). DOI: $10.1021 / \mathrm{jp} 203724 \mathrm{f}$

[27] Psarras G. C., Soto Beobide A., Voyiatzis G. A., Karahaliou P. K., Georga S. N., Krontiras C. A., Sotiropoulos J.: Dielectric and conductivity processes in poly(ethylene terephthalate) and poly(ethylene naphthalate) homopolymers and copolymers. Journal of Polymer Science Part B: Polymer Physics, 44, 3078-3092 (2006). DOI: $10.1002 /$ polb.20939

[28] Schönhals A.: Molecular dynamics in polymer model systems. in 'Broadband dielectric spectroscopy' (eds: Kremer F., Schönhals A.) Springer, Berlin, 225-293 (2003).

[29] Tsangaris G. M., Psarras G. C., Manolakaki E.: DC and $\mathrm{AC}$ conductivity in polymeric particulate composites of epoxy resin and metal particles. Advanced Composites Letters, 8, 25-29 (1999).

[30] Psarras G. C.: Hopping conductivity in polymer matrixmetal particles composites. Composites Part A: Applied Science and Manufacturing, 37, 1545-1553 (2006).

DOI: 10.1016/j.compositesa.2005.11.004

[31] Psarras G. C.: Charge transport properties in carbon black/polymer composites. Journal of Polymer Science Part B: Polymer Physics, 45, 2535-2545 (2007). DOI: $10.1002 /$ polb. 21278

[32] Alig I., Lellinger D., Dudkin S. M., Pötschke P.: Conductivity spectroscopy on melt processed polypropylene-multiwalled carbon nanotube composites: Recovery after shear and crystallization. Polymer, 48, 10201029 (2007).

DOI: $10.1016 /$ j.polymer.2006.12.035 